\title{
Structural Development of Nanosilver on Metal Oxide Nanofibrous Membrane by Plasma Enhanced Chemical Vapor Deposition (PECVD)
}

\author{
Nakarin Subjalearndee, ${ }^{\mathrm{a}}$ Dirk Hegemann, ${ }^{\mathrm{b}}$ Martin Amberg, ${ }^{\mathrm{b}}$ Barbara Hanselmann, ${ }^{\mathrm{b}}$ Patrick \\ Rupper $^{\mathrm{b}}$ and Varol Intasanta $\mathrm{a}^{\mathrm{a}}$ \\ ${ }^{a}$ Nano Functional Textile Laboratory, National Nanotechnology Center, National \\ Science and Technology Development Agency, 111 Phahonyothin Road, Klong Nueng, \\ Klong Luang, Pathumthani, 12120, Thailand. \\ ${ }^{\mathrm{b}}$ Empa, Swiss Federal Laboratories for Materials Science and Technology, Advanced Fibers, \\ Lerchenfeldstrasse 5, St. Gallen, Switzerland \\ Corresponding Author Email: varol@ nanotec.or.th
}

\begin{abstract}
Deposition of functional metal films onto mechanically-sensitive nanostructures has been challenging as conventional wet reduction process could easily lead to physical damages and structural inhomogeneity at nanometer length scale. As PECVD technique not only offers noninvasive approach to single layer metal coating, it also entails processes' scalability and cost-effectiveness. In this study, we investigate the formation of metal nanolayers on visible-light active $\mathrm{ZnWO}_{4}-\mathrm{TiO}_{2}$ nanofibrous membrane as a model substrate against pilotscale PECVD reactor drum's rotating speed as a key parameter at fixed internal pressure and power input. The characteristics of sputtered Ag particles are fully examined by XRD, TEM and XPS techniques. At low rotating speed (less than $0.1 \mathrm{~m} / \mathrm{min}$ ), thick layer of silver is observed with strong antibacterial function. At high speed (more than $18 \mathrm{~m} / \mathrm{min}$ ) silver thin film disappears as silver nanoislands emerge on surface of the metal oxide nanofibrous substrate suggesting nucleation stage of silver deposition. Interestingly, at $6 \mathrm{~m} / \mathrm{min}$, the obtained metal-metal oxide hybrid shows enhanced photocatalytic activity and excellent antibacterial property.
\end{abstract}

Keywords: Plasma enhanced chemical vapor deposition, wet photodeposition, metal oxide nanofiber, silver nanoparticle.

This document is the accepted manuscript version of the following article: Subjalearndee, N., Hegemann, D., Amberg, M., Hanselmann, B., Rupper, P., \& Intasanta, V. (2018). Structural development of nanosilver on metal oxide nanofibrous membrane by plasma enhanced chemical vapor deposition (PECVD). Applied Surface Science, 452, $306-313$. https://doi .org/10.1016/j.apsusc.2018.04.215 


\section{Introduction}

Metal-metal oxide nanofibers and nanofibrous membranes offered functional platforms for advanced material development towards sensing [1, 2] , energy conversion [3, 4], environmental remediation [5, 6], protective textiles [7], etc [8], taking advantages of their unique non-agglomeration nature, high accessible surface area and chemical versatility, in comparison with their nanoparticulate counterparts [9]. However, such conventional metal coating on metal oxide nanofibers as wet photodeposition suffered not only complicated and costly steps [10], but also inhomogeneous deposition quality and unavoidable structural damages during mechanical stirring required in such wet processes. Furthermore, liquid waste from unreacted metal precursor and unbound metal nanocolloids could also raise a serious environmental issue. A solution to such problem required a new approach that would allow delicate, noninvasive and homogeneous metal deposition on the mechanically fragile metal oxide nanofibrous membrane.

Among various coating techniques, chemical vapor deposition (CVD) offers versatile and high quality of deposited polymerized/nanoparticulate thin films in terms of surface homogeneity and good reproducibility [11]. Existing hybrid CVD techniques are classified by their deposition conditions such as atomic layer CVD (ALCVD) [12], low-pressure CVD (LPCVD) [13], photo-initiated CVD (PICVD) [14] or plasma-enhanced CVD (PECVD) [15]. The distinct advantages of PECVD over other CVD techniques are low-temperature requirements, thickness control over large substrate areas and commercial availability for industrial scale production. However, unavoidable drawbacks for this technique include cost associated with high vacuum system for generating plasma which is more expensive than such conventional technique as thermal CVD [16, 17]. Therefore, when considering the facile and cost-effective coating conditions with various possibility of utilizing chemical precursors for nanoparticulate thin film formations, PECVD suits all of primary requirements for nanofibrous membrane coating [18].

In this present contribution, we pioneered the distinctive characteristic of PECVDdeposited Ag as a model noble metal on photocatalytic metal oxide nanofibers with respect to their wet-deposition counterparts. By simple control of drum rotating speed, silver's surface structure ranged from an ultrathin film to nanoparcitulate layer, showing difference in antibacterial and photocatalytic performances of the resulted metal-metal oxide hybrid nanofibrous membranes. The developed nanosilver-decorated metal oxide nanofibrous 
membrane represented a multifunctional all-inorganic nanocatalyst which could be applied to variety of applications such as antibacterial filters, flow-through membranes, liquid filtrations $[5,19]$ and chemical or photo-catalysis $[20,21]$.

\section{Materials and methods}

\subsection{Materials}

Polyvinylpyrrolidone (PVP, $\mathrm{M}_{\mathrm{w}} \sim 1,300,000$, Fluka), ammonium metatungstate hydrate (AMT, $\left(\mathrm{NH}_{4}\right)_{6}-\mathrm{H}_{2} \mathrm{~W}_{12} \mathrm{O}_{40} \cdot \mathrm{xH}_{2} \mathrm{O}$, Sigma Aldrich), zinc acetate dehydrate ( $\mathrm{ZAH}$, $\mathrm{C}_{4} \mathrm{H}_{6} \mathrm{O}_{4} \mathrm{Zn} \cdot 2 \mathrm{H}_{2} \mathrm{O}, \geq 98.0 \%$, Sigma Aldrich), silver nitrate ( $\geq 99.0 \%$, Sigma Aldrich), titanium (IV) isopropoxide solution (TIP, $\mathrm{C}_{12} \mathrm{H}_{28} \mathrm{O}_{4} \mathrm{Ti}, \geq 97.0 \%$, Sigma Aldrich), ethanol (99.8\%, Sigma Aldrich), N,N-dimethylformamide (DMF $\geq 99.0 \%$, Sigma Aldrich) and glacial acetic acid (Sigma Aldrich), were of analytical grade and used as received.

\subsection{Instrumentations}

The physical and chemical characteristics of nanofibers were characterized by scanning electron microscopy (SEM, S3400N, working distance at $5 \mathrm{~mm}$; SEM-EDX, working distance $10 \mathrm{~mm}$ ) and high resolution transmission electron microscopy (HRTEM, JEOL JEM-2010).

The crystal structures of metal oxide nanofibers were determined by X-ray diffraction (XRD) patterns in a range of $2 \theta=15-85^{\circ}$ using an X-ray diffractometer (Bruker, D8 advance) with a $\mathrm{Cu}$ source $\left(\lambda=1.542^{\circ} \mathrm{A}\right)$.

The atomic binding energy and chemical composition at the surface of metal oxide nanofibrous membranes were characterized by X-ray photoelectron spectroscopy (XPS; PHI5000 Versaprobe II, ULVAC-PHI, Japan) under monochromatic Al Ka radiation source at $1486.6 \mathrm{eV}$ at SUT-NANOTEC-SLRI joint research facility, Thailand.

Plasma-enhanced chemical vapor deposition (PECVD) process was performed by employing the plasma CVD roll to roll pilot reactor (Swiss Federal Laboratories for Materials Science and Technology (Empa), Switzerland) [22] . 


\subsection{Preparation of electrospinning precursor solution}

The electrospinning precursor solution was freshly prepared under fume hood. A $10 \% \mathrm{w} / \mathrm{v}$ PVP solution was prepared by dissolving PVP in ethanol (300 ml) under magnetic stirring until the cloudy solution turned colorless. In two separate beakers, $10 \% \mathrm{w} / \mathrm{v}$ AMT solution and $10 \% \mathrm{w} / \mathrm{v}$ ZAH solution were prepared in DMF $(60 \mathrm{ml})$ under magnetic stirring for 20 minutes. Subsequently, the prepared AMT and ZAH solutions were added dropwise into the PVP solution under magnetic stirring, respectively. In the final step, TIP (60 ml) was added into the solution prior to addition of acetic acid $(60 \mathrm{ml})$.

\subsection{Fabrication of nanofibrous membranes via a Nanospider machine}

Nanospider machine (NS LAB 500) was selected for producing nanofibrous membrane via a needle-free electrospinning method. Firstly, the prepared polymer precursor solution $(400 \mathrm{ml})$ was poured into a cylindrical chamber inside the Nanospider machine's chamber. Before performing the electrospinning process, the distance between ground electrode and solution surface was fixed at $180 \mathrm{~mm}$. During the spinning process, voltage was set at $50 \mathrm{kV}$ while the aluminum wire electrode was rotated at $8 \mathrm{rpm}$. Subsequently, the as-spun nanofibrous membrane was calcined in a furnace (Nabertherm $\mathrm{GmbH}$, temperature range: 30-3000 C, model: LT 15/12/P320) under $200 \mathrm{AC}$-processes $\left(200{ }^{\circ} \mathrm{C}\right.$ for an hour then $600{ }^{\circ} \mathrm{C}$ for 4 hours) to remove organic contents while maintaining the physical structure of the metal oxide nanofibrous membrane [23].

\subsection{Ag nanoparticles coating on metal oxide nanofibrous membranes}

\subsubsection{Plasma Enhanced Chemical Vapor Deposition (PECVD) Process}

The coating experiment was performed under the PECVD roll-to-roll pilot scale reactor at Empa [24]. Experimentally, 10 flat metal oxide nanofibrous membranes $(10 \times 10$ $\mathrm{cm}$ ) were attached on commercial cellulose filter papers (Golden Filtech Co., Ltd., Bangkok, Thailand) before fixing them onto the surface of the reactor's drum. Then, the PECVD process was performed by using silver target with controlling pressure and power input at 0.8 $\mathrm{Pa}$ and $600 \mathrm{~W}(360 \mathrm{~V})$, respectively. During sputtering process, Argon (Ar) was applied as sputtering gas at $50 \mathrm{sccm}$. The drum rotating speed was varied at $0.1,6,18$ and $30 \mathrm{~m} / \mathrm{min}$. 


\subsubsection{Wet photodeposition process}

The photodeposition process was performed under visible light irradiation. Three concentrations of silver nitrate solution, $0.3,2.7$ and $5.4 \mathrm{mM}$, were prepared by dissolving silver nitrate in DI water $(60 \mathrm{ml})$. Then nanofibrous membrane $(50 \mathrm{mg})$ was submerged into the silver nitrate solution and magnetically stirred for an hour under visible light irradiation (120 W light bulb) from the light source set $10 \mathrm{~cm}$ apart from the top level of the solution. Finally, the resulting brownish suspension was filtered, washed and dried at $60{ }^{\circ} \mathrm{C}$ for an hour.

\subsection{Antibacterial activity evaluation}

The anti-bacterial activity was performed under dynamic contact according to the standard testing method of ASTM E 2149-10 against E.Coil (ATCC 25922).

\subsection{Methylene blue degradation under visible light}

The photocatalytic activities of the nanofibers were performed against degradation of $\mathrm{MB}$ solution under visible light irradiation (120 W light bulb). Firstly, the metal oxide nanofibers (50 mg) were added into a 5 ppm MB solution $(300 \mathrm{ml})$. Subsequently, the resulting suspension was placed under visible light irradiation with a fix distance between the light source and the top level of solution at $10 \mathrm{~cm}$. Two-ml aliquot of the solution sample was collected, centrifuged and filtered to remove the photocatalyst after every given interval of light irradiation. After 6 hours of the photocatalytic reaction, the concentration of the collected MB solution was measured by UV-Vis spectrophotometer at $663 \mathrm{~nm}$.

\subsection{Methylene blue degradation under natural sunlight}

The field trial photocatalytic activity evaluation of metal oxide nanofibers was performed under natural sunlight irradiation against $5 \mathrm{ppm}$ MB solution (natural sunlight intensity was measured by Lux meter: Heavy duty light meter, Extech instrument, model: 407026). MB solution containing the photocatalyst was prepared by adding nanofibers $(50 \mathrm{mg})$ into a 5 
ppm MB solution $(300 \mathrm{ml})$. Then, the prepared suspension was put on the magnetic stirrer under direct sunlight irradiation. After every given interval of light irradiation, a 1-ml aliquot of the sample was collected, centrifuged and filtered to remove the photocatalyst. Finally, the concentration of MB was measured using a UV-Vis spectrophotometer as mentioned above.

\section{Results and discussion}

\subsection{Sputtered $\mathrm{Ag}^{0}$ nanoparticle characterization}

In this study, we aimed to compare the physical and chemical characteristics of deposited Ag on the metal oxide nanofibers from two different deposition processes-wet photodeposition and PECVD. In addition, we also investigated the effect of drum rotating speed on the characteristics of the Ag layer under PECVD process followed by the studies of anti-bacterial and photocatalytic performances of the resulted hybrids nanofibers.

Synthesis and characterization of $\mathrm{ZnWO}_{4} /$ mixed-phased $\mathrm{TiO}_{2}$ nanofibers $\left(\mathrm{ZnWO}_{4}{ }^{-}\right.$ $\mathrm{TiO}_{2}$ nanofibers) were reported previously [23]. The original structure of metal oxide nanofibers containing $\mathrm{ZnWO}_{4}$ nanorods at the surface is shown in Figure 1a. After performing $\mathrm{Ag}$ photodeposition process with $0.3 \mathrm{mM}$ silver nitrate solution by visible light irradiation, the physical characteristics of the metal oxide nanofibers remained intact (Figure 1b). Under TEM and HRTEM, small sphere-like particles were observed along the surface of the nanofiber (Figure 1c and 1d). The respective diffraction spacings represented (111) planes of metal silyer $\left(\mathrm{Ag}^{0}\right)$ (Figure 1e). In addition, the SAED measurement illustrated the diffraction patterns of (200) planes of $\mathrm{Ag}^{0}$ (Figure 1f). Finally, TEM-EDX spectrum confirmed the presence of silver element on the sample (Figure 1g). 


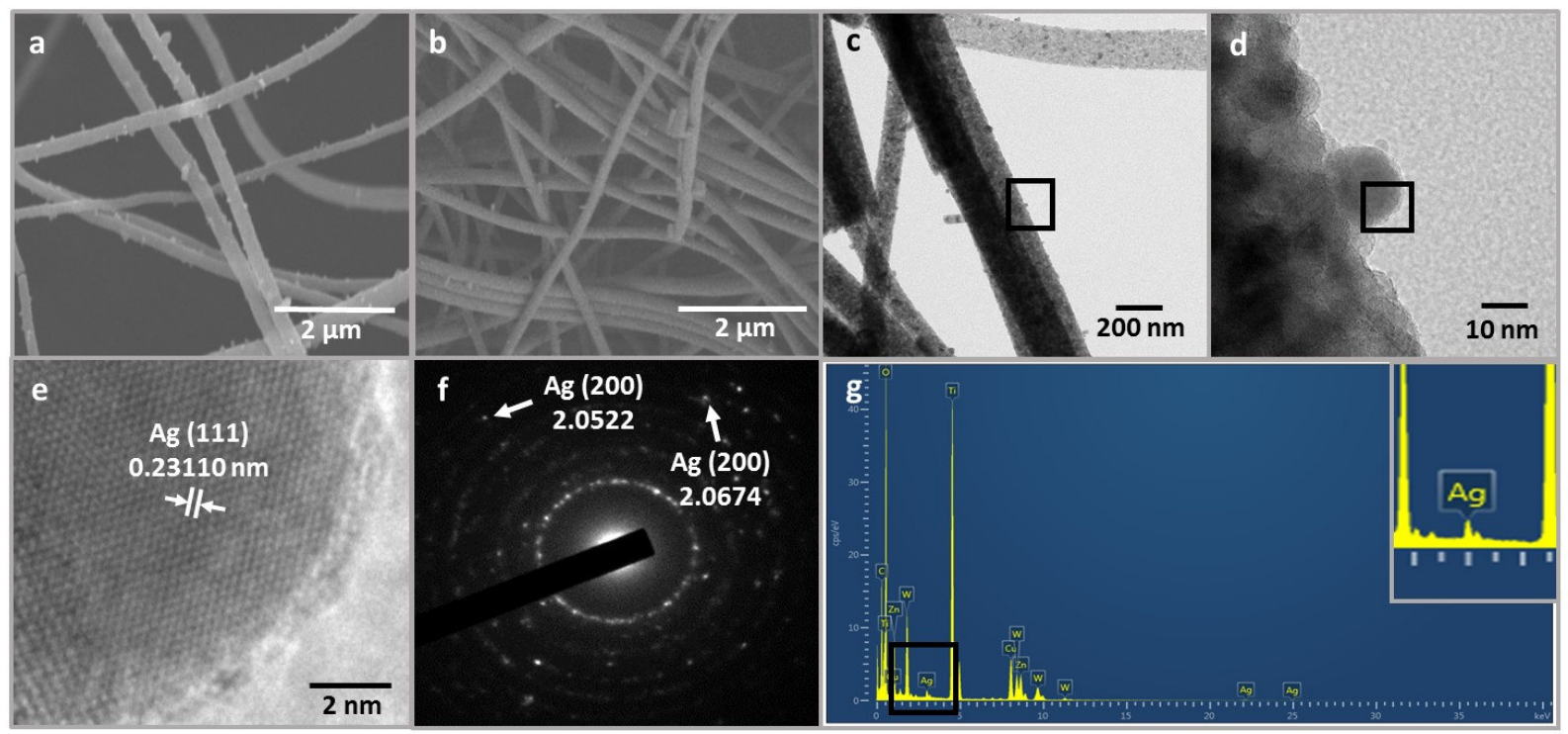

Figure 1. SEM images of $\mathrm{ZnWO}_{4}-\mathrm{TiO}_{2}$ nanofibers (a) before and (b) after wet photodeposition process with $0.3 \mathrm{mM}$ silver nitrate solution (Ag_Dep_0.3 mM). (C) TEM, (d and e) HRTEM, (f) SAED pattern and (g) TEM-EDX spectrum of Ag_Dep_0.3 mM nanofibers.

For the Ag-PECVD process at the lowest drum's rotating speed, the resulted hybrid nanofibers showed homogeneous and similar physical structure to the original metal oxide nanofibers (Figure 2a). SEM-EDX spectrum confirmed the existence of Ag element on the nanofibers (Figure 2c) whereas elemental mapping suggested homogeneous distribution of the element on the nanofibers (Figure 2b). Under TEM, thick nanolayer of nanoparticles was shown along the surface contour of the nanofibers (Figure 2d and 2e). Under HRTEM, (200) planes of $\mathrm{Ag}^{0}$ crystal (Figure $2 \mathrm{f}$ ) were unveiled. Moreover, TEM diffraction pattern also suggested the (200) planes of $\mathrm{Ag}(0)$ as well (Figure 2g). In addition, XRD diffraction patterns are presented in the supporting information (Figure S1). After increasing the drum rotating speed to $6 \mathrm{~m} / \mathrm{min}$, physical characteristics of $\mathrm{Ag}^{0}$ on the nanofibers was also exposed by SEM and HRTEM (Figure S2). It was noticed that the Ag signal under SEM-EDX (Figure S3) was relatively weak, suggesting lower amount of metal on nanofibers at high speed. 


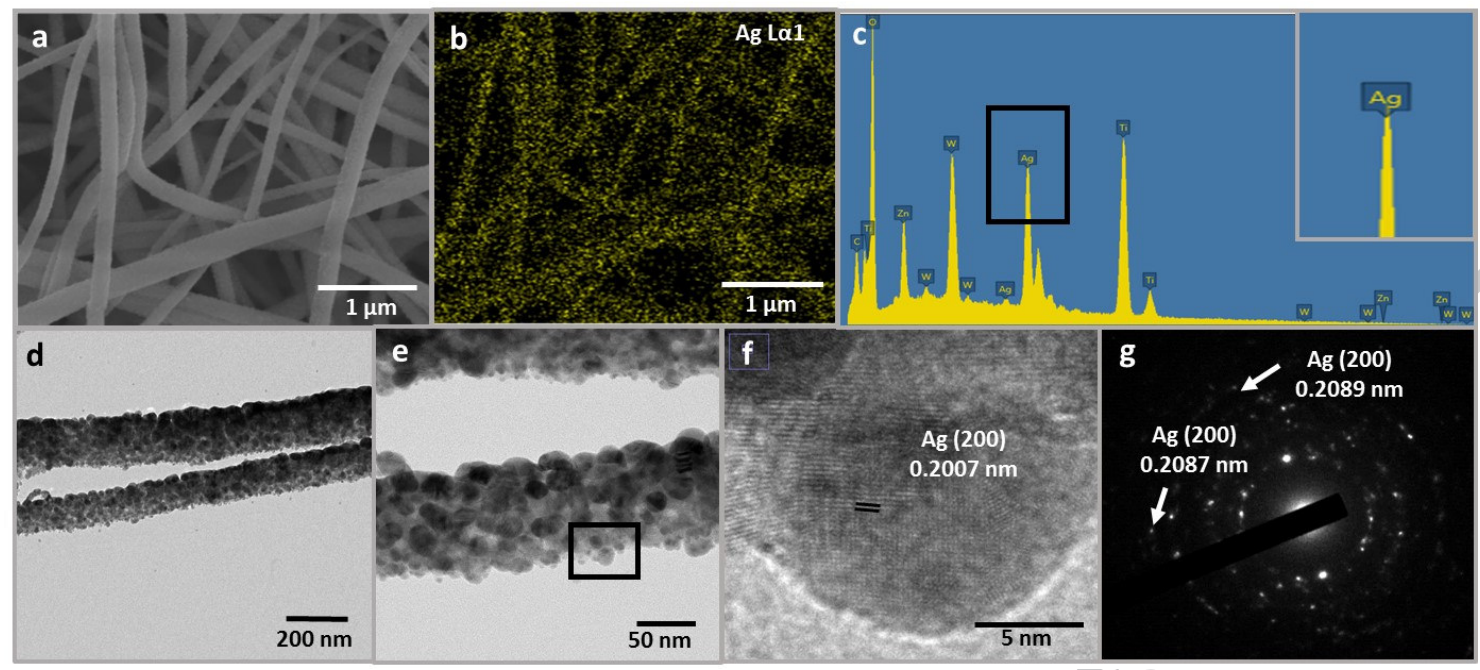

Figure 2. (a) SEM image of $\mathrm{ZnWO}_{4}-\mathrm{TiO}_{2}$ nanofibers after $\mathrm{Ag}$ sputtering at drum rotating speed of $0.1 \mathrm{~m} / \mathrm{min}\left(\mathrm{Ag}_{-} S p u \_0.1\right)$. (b) Ag elemental mapping, (c) SEM-EDX, (d-f) TEM/HREM and (g) SAED pattern of Ag_Spu_0.1 nanofibers.

For process comparison, we employed three different Ag solution concentrations, 0.3, 2.7 and $5.4 \mathrm{mM}$, for wet photo-deposition process before observing the deposited $\mathrm{Ag}$ characteristics on the metal oxide nanofiber by TEM. After the photoreaction, increasing silver nitrate concentration resulted in not only increased Ag content but also propensity of the generated nanoparticles to agglomerate on the surface of nanofibers (Figure 3a-c). For the Ag-PECVD at the slowest speed $(0.1 \mathrm{~m} / \mathrm{min})$, large amount of Ag nanoparticles were observed along the nanofiber surface but no agglomeration or cluster of Ag nanoparticles were found as from the wet photodeposition process (Figure 3d). At moderate speed (6 $\mathrm{m} / \mathrm{min}$ ), fewer $\mathrm{Ag}$ nanoparticles were generated as a single and homogeneous layer along the surface (Figure 3e). At high speed (18 m/min), no Ag nanoparticles were observed (Figure 3f), as the fast moving drum might have interrupted nucleation of metal nanosilver. To elucidate the disappearance of the nanosilver SEM-EDX elemental mapping was performed as illustrated in Figure 3g-i. The results suggested that no silver element nor nanoparticles were observed at the nanofiber surface, while $\mathrm{W}, \mathrm{Zn}, \mathrm{Ti}, \mathrm{O}$ and $\mathrm{C}$ elements were clearly observed (Figure $3 \mathrm{~h}$, inset). In additional to the physical difference at nanometer length scale, the two deposition processes also made their difference at the macroscopic level. Firstly, the appearance of the original metal oxide nanofibrous membrane was shown in Figure 3j. After performing Ag-PECVD, the nanomembrane turned brownish as its 
dimensional integrity remained intact (Figure 3k). However, the wet deposition process transformed the one membrane into countless pieces (Figure 31) possibly due to the mechanical perturbation associated with stirring during the wet reaction. Therefore, it could be concluded that wet photodeposition process was not a suitable process for depositing thin layer of $\mathrm{Ag}$ nanoparticles on mechanically-sensitive metal oxide nanofibrous membrane. Finally, PECVD could be successfully utilized as a noninvasive approach to decorate mechanically-sensitive materials with single and homogeneous layer of metal nanostructures as shown in the Ag model above.

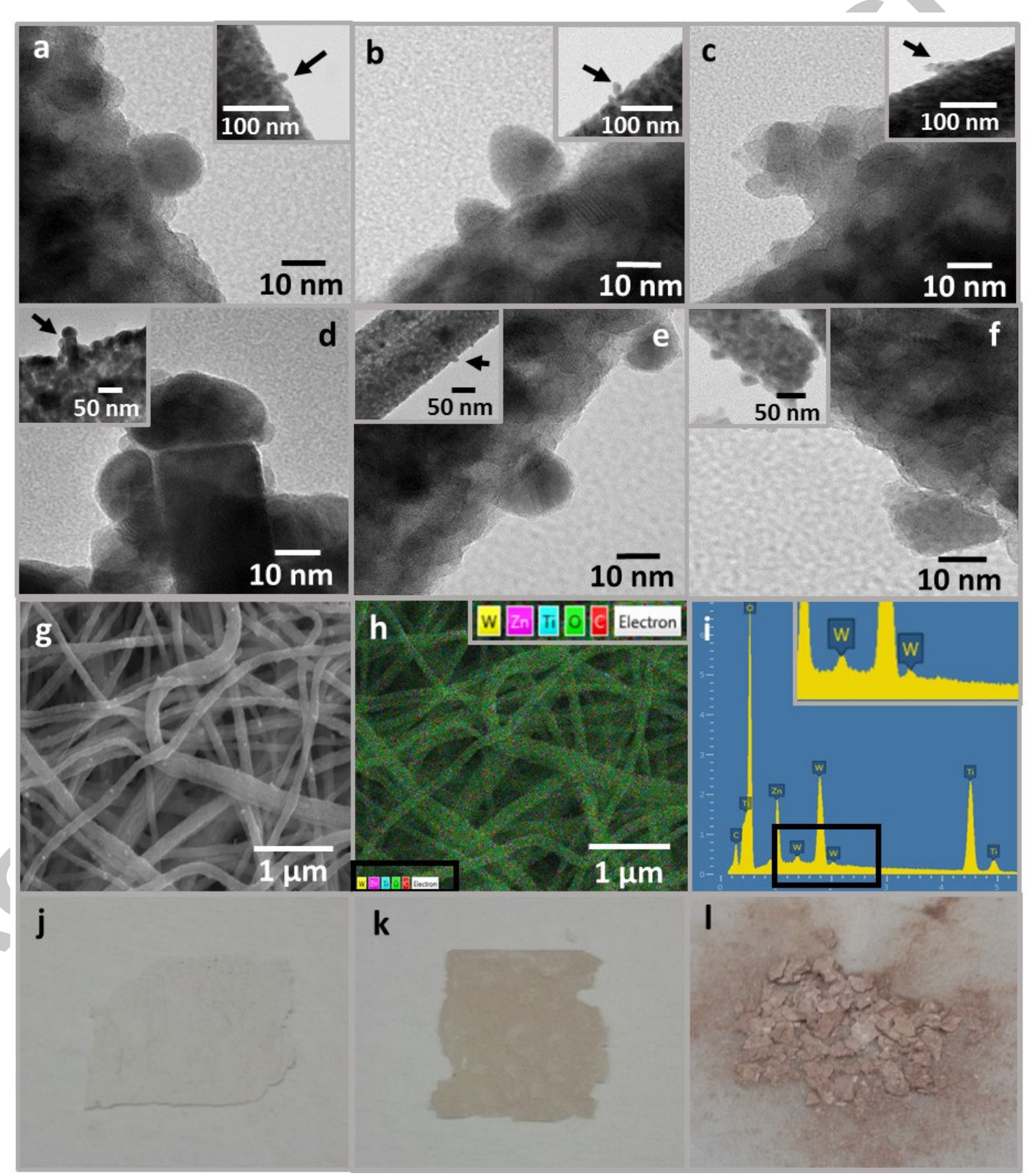


Figure 3. $\mathrm{SEM}$ images of $\mathrm{Ag}$ deposited on $\mathrm{ZnWO}_{4}-\mathrm{TiO}_{2}$ nanofibers by wet photodeposition with (a) 0.3 , (b) 2.7 and (c) $5.4 \mathrm{mM}$ silver nitrate concentration. SEM images of Agsputtered $\mathrm{ZnWO}_{4}-\mathrm{TiO}_{2}$ nanofibers by using drum rotating speed of (d) 0.1 , (e) 6 and (f) 18 m/min. (g) SEM, (h) SEM-EDX elemental analysis and (i) SEM-EDX spectrum of Ag_Spu_18 nanofibers. Pictures of $\mathrm{ZnWO}_{4}-\mathrm{TiO}_{2}$ nanofibrous membrane (j) before and after (k) performing Ag-PECVD and (l) wet photodeposition.

Additionally, a Ag-PECVD experiment at $30 \mathrm{~m} / \mathrm{min}$ was performed in order to investigate the effective of speed on nucleation. After sputtering, the nanofiber showed no Ag structures nor Ag element by SEM/SEM-EDX (Figure 4a-b) or by TEM/TEM-EDX (Figure 4c-e). This could be implied that high drum rotating speed (more than $18 \mathrm{~m} / \mathrm{min}$ ) was relatively fast compared to Ag element's resident time prior to nucleation on surface.

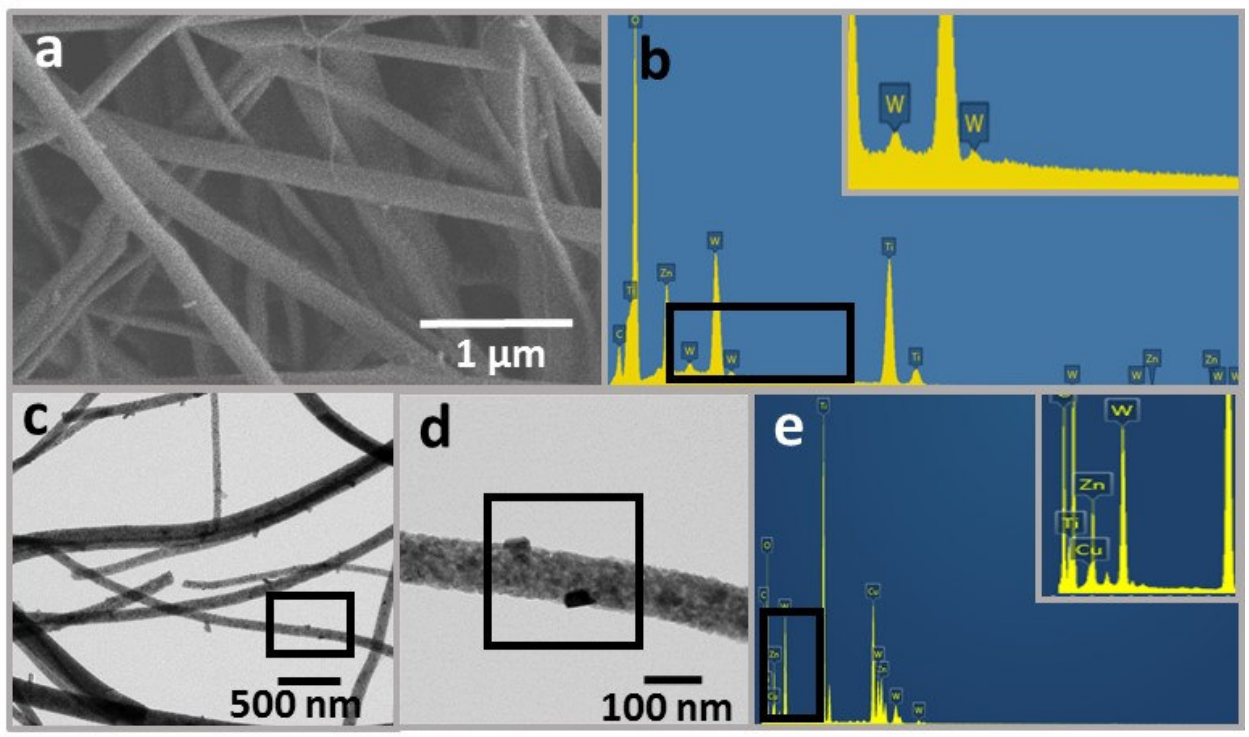

Figure 4. (a) SEM image and (b) SEM-EDX spectrum of Ag_Spu_30 nanofibers. (c-e) TEM, HREM and TEM-EDX spectrum of Ag_Spu_30 nanofibers. 


\subsection{X-ray photoelectron spectroscopy (XPS) analysis}

The XPS survey scan was performed in order to compare the chemical composition between the non-sputtered and Ag sputtered metal oxide nanofibers (Figure 5). From the spectrum, Ag peaks were observed solely at the surface of Ag_Spu_0.1 nanofibers due to presence of thick $\mathrm{Ag}$ nanoparticle layer coated by using slow drum rotating speed. In addition, high intensity of $\mathrm{Ag} 3 \mathrm{~d} 3$ and $\mathrm{Ag} 3 \mathrm{~d} 5$ peaks could be clearly observed at the surface of Ag_Spu_0.1 and Ag_Spu_6 nanofibers along with other chemical compositions of $\mathrm{ZnWO}_{4}-\mathrm{TiO}_{2}$ nanofibers ( $\mathrm{Zn}, \mathrm{W}$ and $\mathrm{O}$ peaks). On the other hand, trace amount of $\mathrm{Ag}$ composition was observed on Ag_Spu_18 nanofibers while the surface of Ag_Spu_30 and $\mathrm{ZnWO}_{4}-\mathrm{TiO}_{2}$ nanofibers showed no Ag content. The primary XPS survey scan strongly agreed with the TEM-EDX analysis, confirming full coverage of Ag particles on the surface of Ag_Spu_0.1 nanofibers.

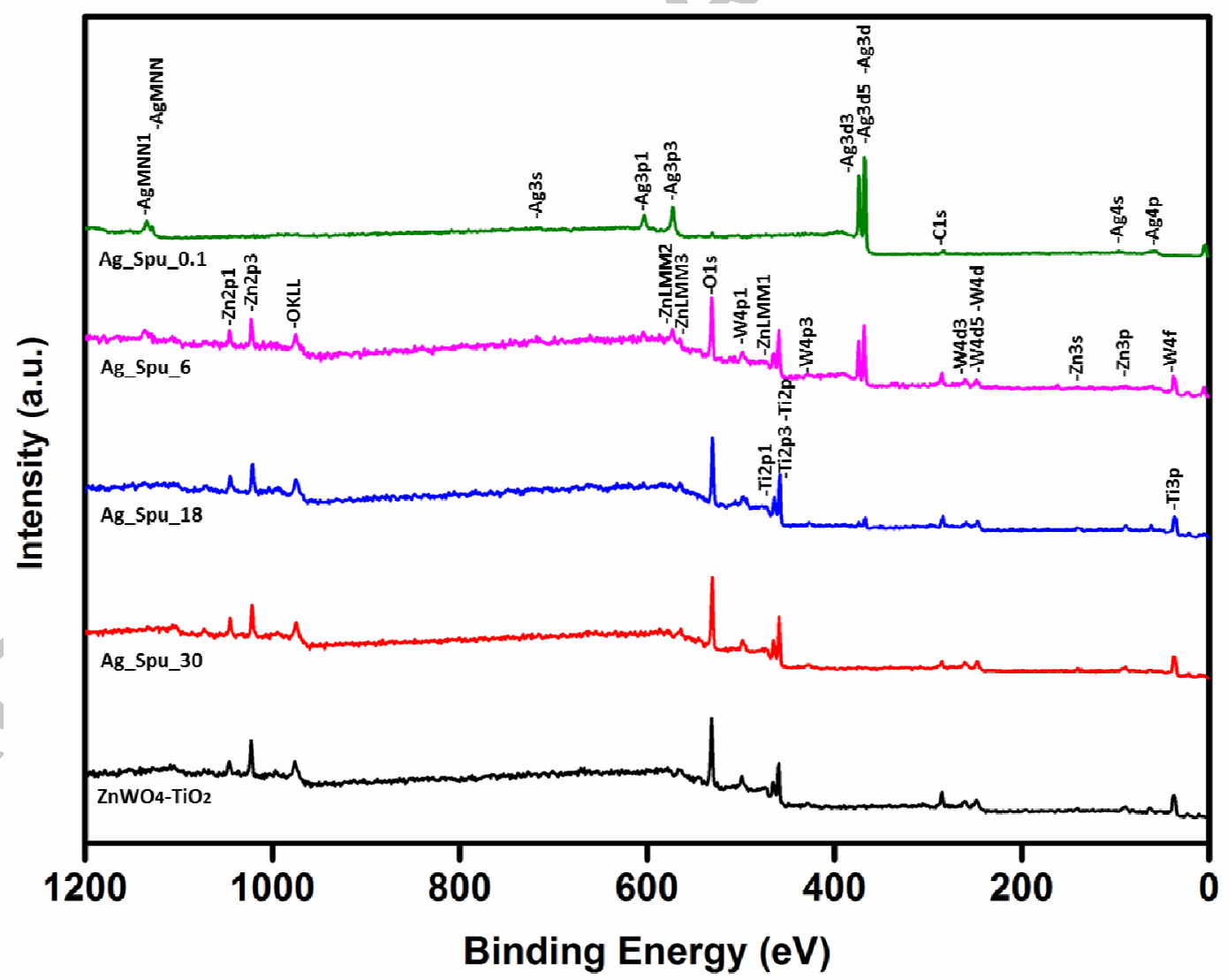

Figure 5. XPS survey scan spectrum of non-sputtered $\mathrm{ZnWO}_{4}-\mathrm{TiO}_{2}, \mathrm{Ag}$ _Spu_0.1, Ag_Spu_6, Ag_Spu_18 and Ag_Spu_30 nanofibrous membranes. 
Furthermore, high resolution XPS analysis of Ag 3d peaks was performed in order to characterize the binding energy of Ag particles on the metal oxide substrate (Figure 6). In the first step, all binding energies of chemical composition were calibrated against residual $\mathrm{C}$ atom $(\mathrm{C} 1 \mathrm{~s}=285 \mathrm{eV})$. After peak fitting by applying the Shirley background, the chemical shift of $\mathrm{Ag} 3 \mathrm{~d}$ peaks was observed in the $\mathrm{Ag}$ sputtered metal oxide nanofibers using slow drum rotating speed. At $0.1 \mathrm{~m} / \mathrm{min}$ speed, loss feature peaks were observed at $372.8 \mathrm{eV}$ and $378.7 \mathrm{eV}$ while $\mathrm{Ag} \mathrm{3} \mathrm{d}_{5 / 2}$ peak was observed at $369.1 \mathrm{eV}$ (Figure 6a). A chemical shift of Ag atom at the surface of Ag_Spu_0.1 nanofibers was observed at the higher binding energy region due to the influence of atoms' structural arrangement and particle size $[25,26]$. It was noted that, at moderate and fast sputtering speed $(6,18$ and $30 \mathrm{~m} / \mathrm{min}), \mathrm{Ag} 3 \mathrm{~d}_{5 / 2}$ peaks observed at $368.0 \mathrm{eV}, 367.7 \mathrm{eV}$ and $367.6 \mathrm{eV}$, respectively, (Figure 6b-d) represented the metallic characteristics of $\mathrm{Ag}[27,28]$. Moreover, the relatively low peak intensity in high resolution XPS spectra of Ag_Spu_18 and Ag_Spu_30 nanofibers hinted for trace amount of Ag composition at the nanofiber surfaces, which was in agreement with the Ag signal results from SEM-EDX characterization. It could be said that applying fast drum rotating speed results in low Ag particle nucleation on the metal oxide nanofiber surface.
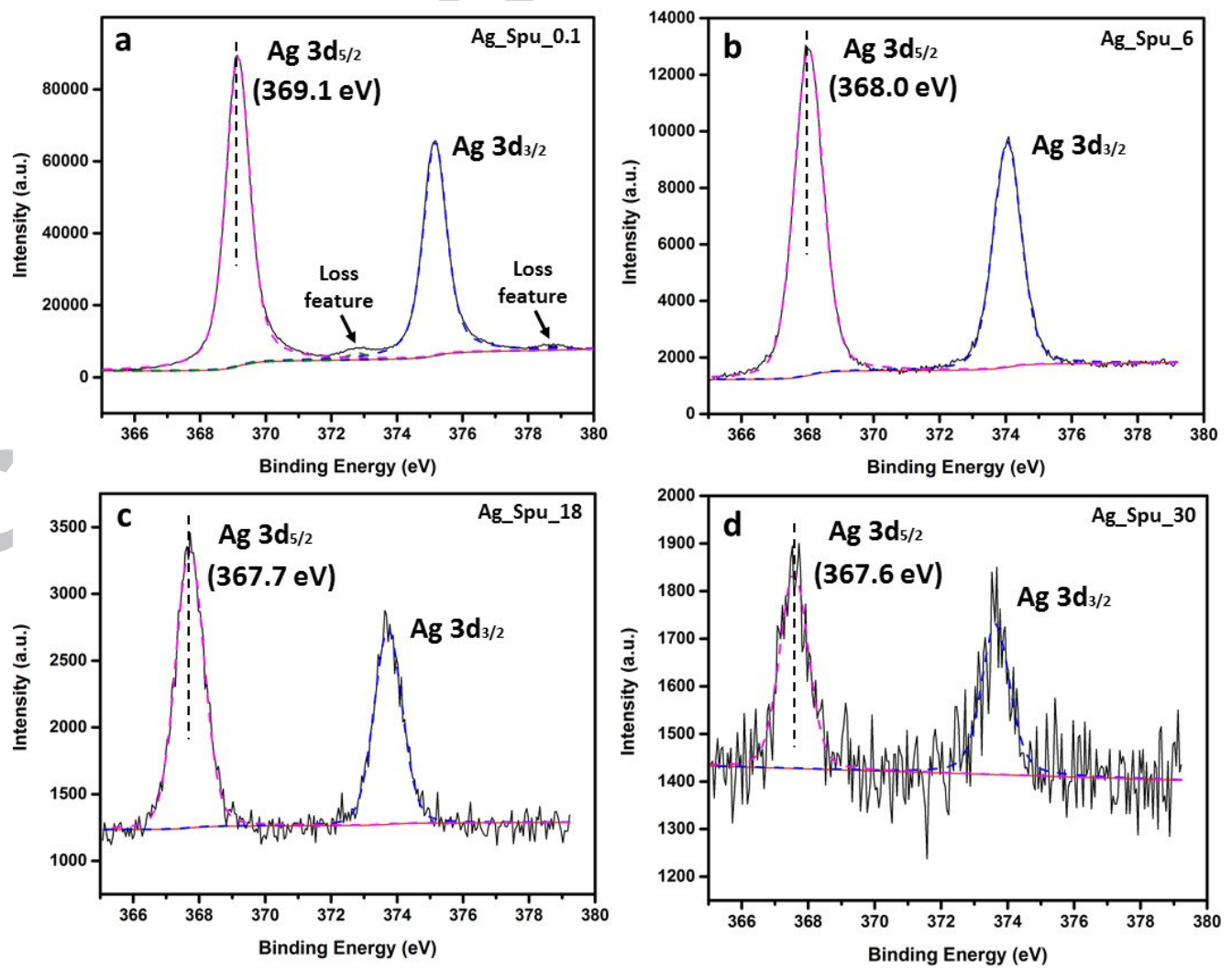
Figure 6. XPS analysis of Ag3d core level spectra of (a) Ag_Spu_0.1, (b) Ag_Spu_6, (c) Ag_Spu_28 and (d) Ag_Spu_30 nanofibrous membranes (raw data: black line; fitted Ag3d5/2 peak: pink dashed line; fitted Ag3d3/2 peak: blue dashed line).

\subsection{Anti-bacterial evaluation}

The original form of nanofibers showed no significant antibacterial efficiency under standard testing method of ASTM E 2149-10. Similarly, the Ag-sputtered nanofibers from drum rotating speed of 18 and $30 \mathrm{~m} / \mathrm{min}$ also unveiled sparse antibacterial activity of $15.36 \%$ and $0 \%$, respectively. These results were also in agreement with the above disappearance of nucleated silver nanodomains on the nanofibers' surface. In contrast, both Ag-sputtered nanofibers from lower drum rotating speed of 6 and $0.1 \mathrm{~m} / \mathrm{min}$ presented exceptional antibacterial activities of more than $99.99 \%$ (Figure 7). This drastic functional improvement confirmed that those prevalent silver nanodomains on surface were highly-active antibacterial agent. This could imply that moderate drum rotating speed of $6 \mathrm{~m} / \mathrm{min}$ was sufficient to generate a single layer of nanostructured silver layer with an excellent antibacterial performance.

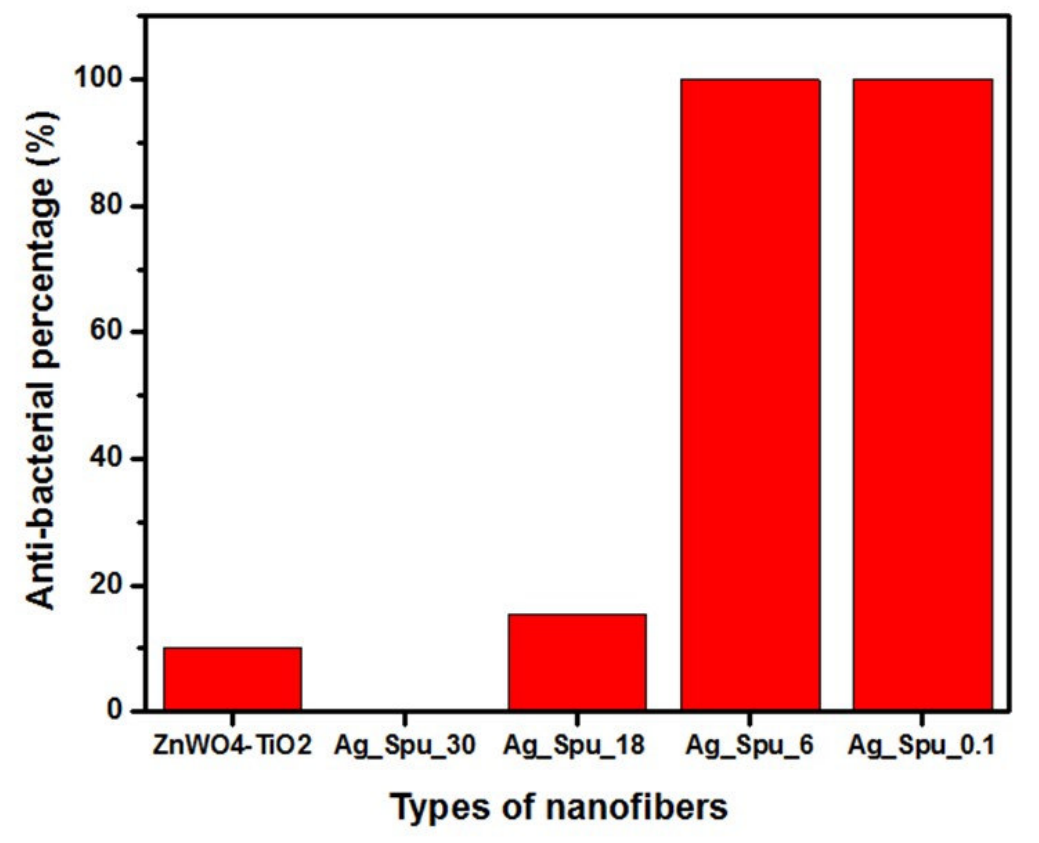


Figure 7. Antibacterial result of $\mathrm{Ag}$ sputtered $\mathrm{ZnWO}_{4}-\mathrm{TiO}_{2}$ nanofibers by $\mathrm{Ag}-\mathrm{PECVD}$ process at different drum rotating speeds.

\subsection{Photocatalytic evaluation}

From the antibacterial activity result, Ag_Spu_6 and Ag_Spu_0.1 nanofibers showed excellent activity in comparison with the uncoated nanofiber. In this experiment, we selected both nanofibers for further evaluating photocatalytic performance under visible and natural sunlight irradiation. Under visible light irradiation, $\mathrm{ZnWO}_{4}-\mathrm{TiO}_{2}$ nanofibers were selected as a control. After 6 hours of photoreaction, Ag_Spu_6 nanofibers showed the better photocatalytic activity against degradation of $5 \mathrm{ppm} \mathrm{MB}$ in comparison with that of $\mathrm{ZnWO}_{4}$ $\mathrm{TiO}_{2}$ and Ag_Spu_0.1 nanofibers (Figure S4, supporting information). Interestingly, at the first hour of the photoreaction, $\mathrm{ZnWO}_{4}$ and $\mathrm{Ag} \_\mathrm{Spu}$-0.1 nanofibers presented similar photocatalytic activity. However, at the end of the photoreaction, the control nanofibers performed better than Ag_Spu_0.1 nanofiber as evident by its relatively less MB absorbance. It could be inferred from this result that the thick layer of surface-deposited Ag nanoparticles manifested into partial blockade preventing the light irradiation to reach the surface's photocatalytic sites, reducing the materials' photocatalytic potential.

Moreover, the photocatalytic activity evaluation against natural sunlight was performed on a sunny day, with an average sunlight intensity, measured by a lux meter every 2 hours throughout the photoreaction, close to 36,100 Lux (Figure 8). The comparison of fading MB colors before and after lengthened reaction was also shown. From the pictures, it could be clearly seen that all photocatalysts showed excellent MB elimination under sunlight irradiation by converting blue solution into colorless solution. Evidently, after 30 minutes of the reaction, all catalysts showed similar performance against MB degradation. However, the Ag_Spu_6 nanofiber revealed the highest activities after 3 and 6 hours. In particular, after 3 hours of the reaction time, the nanofiber showed MB absorbance at 0.234 in comparison with 0.305 and 0.273 of Ag_Spu_0.1 and $\mathrm{ZnWO}_{4}-\mathrm{TiO}_{2}$ nanofibers, respectively. At the end of the reaction, the Ag_Spu_6 also presented the highest photocatalytic activities with the MB absorbance at 0.039 compared to 0.047 for both $\mathrm{Ag} \_\mathrm{Spu} \_0.1$ and $\mathrm{ZnWO}_{4}-\mathrm{TiO}_{2}$ nanofibers. 

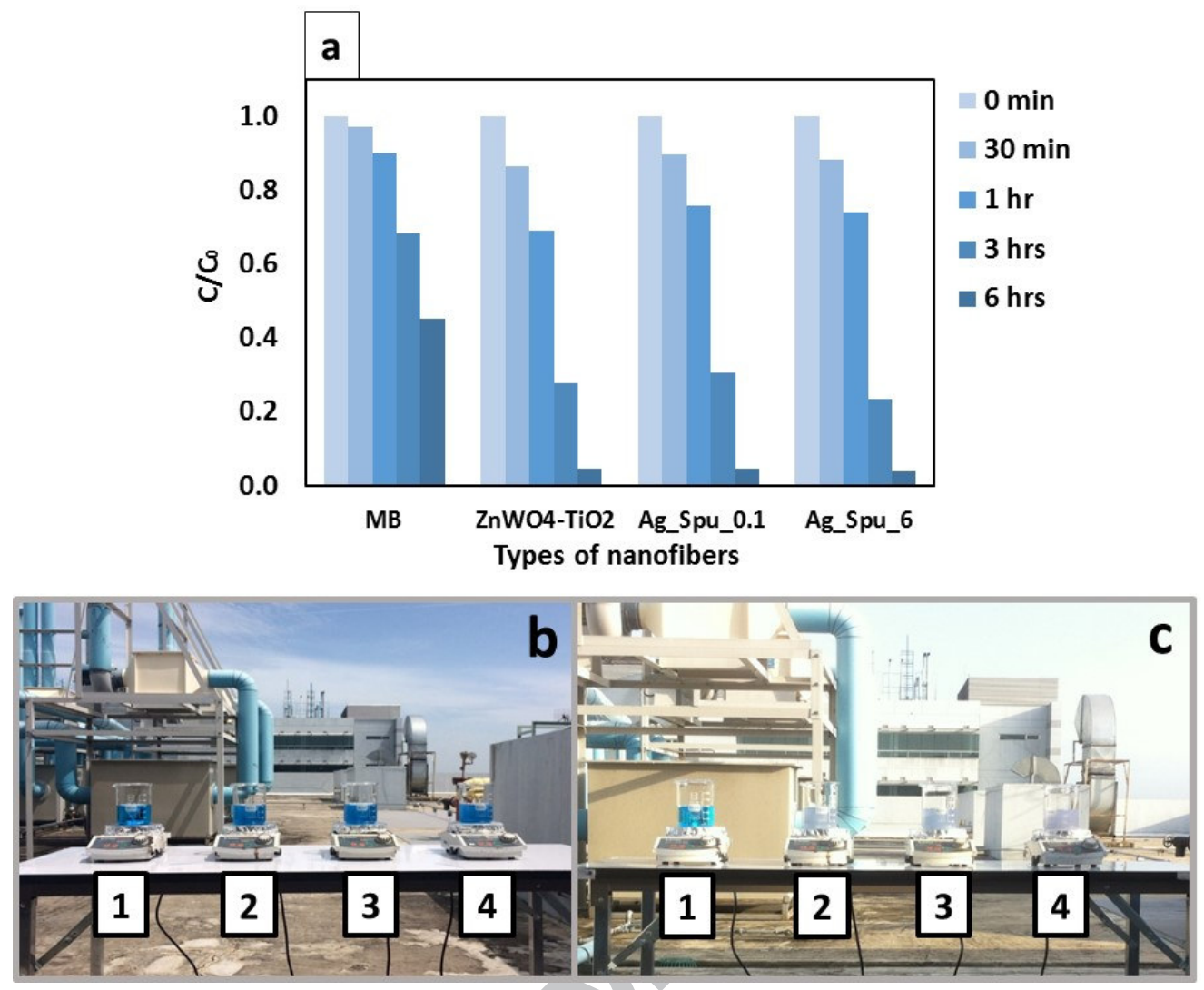

Figure 8. (a) Photocatalytic activity of Ag sputtered nanofibers against 5 ppm MB solution under natural sunlight. Pictures of photocatalytic activity evaluation experiment under natural sunlight irradiation (b) in the beginning and (c) end of the experiment ( $1=\mathrm{MB}$ solution, $2=$ Ag_Spu_0.1, 3=Ag_Spu_0.6 and 4= $\mathrm{ZnWO}_{4}-\mathrm{TiO}_{2}$ ).

\section{Conclusion}

Plasma enhanced chemical vapor deposition (PECVD) technique was successfully demonstrated as noninvasive means to deposit structurally tunable homogeneous metal nanolayer on mechanically-sensitive metal oxide nanofibrous membrane. Unique characteristics and presence of sputtered Ag nanoparticles by different drum rotating speeds were characterized by XRD, TEM-EDX and HRTEM. In addition, high resolution XPS spectra also confirmed the characteristics of metallic Ag particles at the surface of $\mathrm{ZnWO}_{4}$ $\mathrm{TiO}_{2}$ nanofibers which was in agreement with the primary characterization techniques. As visible-light active $\mathrm{ZnWO}_{4}-\mathrm{TiO}_{2}$ nanofibrous membrane was utilized as a model functional substrate, thick nanolayer of silver with potent antibacterial function was obtained at low 
rotating PECVD's drum speed (less than $0.1 \mathrm{~m} / \mathrm{min}$ ). At high speed (more than $18 \mathrm{~m} / \mathrm{min}$ ), the silver layer emerged as nucleating islands on the surface of metal oxide nanofibrous substrate. At optimized speed of $6 \mathrm{~m} / \mathrm{min}$, the resulted metal-metal oxide nanofibrous hybrid showed enhanced photocatalytic activity and excellent antibacterial property.

\section{Acknowledgements}

The authors would like to thank the Thailand Research Fund (Grant Number RSA5780067) and National Nanotechnology Center for financial support and the Swiss Federal Laboratories for Materials Science and Technology (Empa) for pilot-scale plasma enhanced chemical vapor deposition (PECVD) experiments. 


\section{References}

[1] D.R. Miller, S.A. Akbar, P.A. Morris, Nanoscale metal oxide-based heterojunctions for gas sensing: A review, Sensors and Actuators B-Chemical, 204 (2014) 250-272.

[2] K. Mondal, A. Sharma, Recent advances in electrospun metal-oxide nanofiber based interfaces for electrochemical biosensing, RSC Adv., 6 (2016) 94595-94616.

[3] M. Tanaka, Development of ion conductive nanofibers for polymer electrolyte fuel cells, Polym. J., 48 (2016) 51-58.

[4] C. Wang, X.J. Li, Z.Q. Cai, J. Huang, X. Fan, H. Liu, W.L. Xu, D. Fang, A Review on the Electrospun Oxide Nanofibers for Anode Electrodes in Lithium-Ion Batteries, Curr. Nanosci., 13 (2017) 394-409.

[5] S. Homaeigohar, M. Elbahri, Nanocomposite Electrospun Nanofiber Membranes for Environmental Remediation, Materials, 7 (2014) 1017-1045.

[6] V. Thavasi, G. Singh, S. Ramakrishna, Electrospun nanofibers in energy and environmental applications, Energy Environ. Sci., 1 (2008) 205-221.

[7] S. Sundarrajan, A.R. Chandrasekaran, S. Ramakrishna, An Update on Nanomaterials-Based Textiles for Protection and Decontamination, J. Am. Ceram. Soc., 93 (2010) 3955-3975.

[8] J.J. Xue, J.W. Xie, W.Y. Liu, Y.N. Xia, Electrospun Nanofibers: New Concepts, Materials, and Applications, Accounts of Chemical Research, 50 (2017) 1976-1987.

[9] M.C. Daniel, D. Astruc, Gold nanoparticles: Assembly, supramolecular chemistry, quantum-sizerelated properties, and applications toward biology, catalysis, and nanotechnology, Chemical Reviews, 104 (2004) 293-346.

[10] R. Camposeco, S. Castillo, I. Mejía-Centeno, J. Navarrete, J. Marín, Characterization of physicochemical properties of $\mathrm{Pd} / \mathrm{TiO} 2$ nanostructured catalysts prepared by the photodeposition method, Materials Characterization, 95 (2014) 201-210.

[11] S. Vallejos, F. Di Maggio, T. Shujah, C. Blackman, Chemical Vapour Deposition of Gas Sensitive Metal Oxides, Chemosensors, 4 (2016) 18.

[12] C. Guan, J. Wang, Recent Development of Advanced Electrode Materials by Atomic Layer Deposition for Electrochemical Energy Storage, Adv. Sci., 3 (2016) 23.

[13] K. Teii, Plasma Deposition of Diamond at Low Pressures: A Review, IEEE Trans. Plasma Sci., 42 (2014) 3862-3869.

[14] C.A.D. Dion, J.R. Tavares, Photo-initiated chemical vapor deposition as a scalable particle functionalization technology (a practical review), Powder Technol., 239 (2013) 484-491.

[15] M.C. Vasudev, K.D. Anderson, T.J. Bunning, V.V. Tsukruk, R.R. Naik, Exploration of PlasmaEnhanced Chemical Vapor Deposition as a Method for Thin-Film Fabrication with Biological Applications, ACS Applied Materials \& Interfaces, 5 (2013) 3983-3994.

[16] K.L. Choy, Chemical vapour deposition of coatings, Prog. Mater. Sci., 48 (2003) 57-170.

[17] S.R. Droes, T.T. Kodas, M.J. Hampden-Smith, Plasma-Enhanced Chemical Vapor Deposition (PECVD), in: A.W. Weimer (Ed.) Carbide, Nitride and Boride Materials Synthesis and Processing, Springer Netherlands, Dordrecht, 1997, pp. 579-603.

[18] G. Malandrino, Chemical Vapour Deposition. Precursors, Processes and Applications. Edited by Anthony C. Jones and Michael L. Hitchman, Angewandte Chemie International Edition, 48 (2009) 7478-7479.

[19] C.M. Chew, M.K. Aroua, M.A. Hussain, Advanced process control for ultrafiltration membrane water treatment system, Journal of Cleaner Production, 179 (2018) 63-80.

[20] H. Koga, H. Ishihara, T. Kitaoka, A. Tomoda, R. Suzuki, H. Wariishi, NOX reduction over paperstructured fiber composites impregnated with Pt/Al2O3 catalyst for exhaust gas purification, Journal of Materials Science, 45 (2010) 4151-4157.

[21] M. Modesti, M. Roso, C. Boaretti, S. Besco, D. Hrelja, P. Sgarbossa, A. Lorenzetti, Preparation of smart nano-engineered electrospun membranes for methanol gas-phase photoxidation, Applied Catalysis B-Environmental, 144 (2014) 216-222.

[22] D. Hegemann, Plasma polymerization and its applications in textiles, 2006. 
[23] N. Subjalearndee, V. Intasanta, Thermal relaxation in combination with fiberglass confined interpenetrating networks: a key calcination process for as-desired free standing metal oxide nanofibrous membranes, RSC Adv., 6 (2016) 86798-86807.

[24] D. Hegemann, B. Hanselmann, N. Blanchard, M. Amberg, Plasma-Substrate Interaction during Plasma Deposition on Polymers, Contrib. Plasma Phys., 54 (2014) 162-169.

[25] J. Radnik, C. Mohr, P. Claus, On the origin of binding energy shifts of core levels of supported gold nanoparticles and dependence of pretreatment and material synthesis, Physical Chemistry Chemical Physics, 5 (2003) 172-177.

[26] A.A. Mosquera, J.M. Albella, V. Navarro, D. Bhattacharyya, J.L. Endrino, Effect of silver on the phase transition and wettability of titanium oxide films, Sci Rep, 6 (2016) 14.

[27] Y.-Y. Chang, Y.-N. Shieh, H.-Y. Kao, Optical properties of $\mathrm{TiO}$ thin films after Ag ion implantation, Thin Solid Films, 519 (2011) 6935-6939.

[28] D. Briggs, Handbook of X-ray Photoelectron Spectroscopy C. D. Wanger, W. M. Riggs, L. E. Davis, J. F. Moulder and G. E.Muilenberg Perkin-Elmer Corp., Physical Electronics Division, Eden Prairie, Minnesota, USA, 1979. 190 pp. \$195, Surface and Interface Analysis, 3 (1981) v-v. 


\section{Structural Development of Nanosilver on Metal Oxide Nanofibrous Membrane by}

\section{Plasma Enhanced Chemical Vapor Deposition (PECVD)}

Nakarin Subjalearndee, ${ }^{\mathrm{a}}$ Dirk Hegemann, ${ }^{\mathrm{b}}$ Martin Amberg, ${ }^{\mathrm{b}}$ Barbara Hanselmann, ${ }^{\mathrm{b}}$ Patrick Rupper $^{\mathrm{b}}$ and Varol Intasanta ${ }^{\mathrm{a} *}$

${ }^{\mathrm{a} N a n o}$ Functional Textile Laboratory, National Nanotechnology Center, National

Science and Technology Development Agency, 111 Phahonyothin Road, Klong Nuéng,

Klong Luang, Pathumthani, 12120, Thailand.

${ }^{\mathrm{b}}$ Empa, Swiss Federal Laboratories for Materials Science and Technology, Advanced Fibers, Lerchenfeldstrasse 5, St. Gallen, Switzerland

Corresponding Author Email: varol@ nanotec.or.th

\section{Graphical Abstract}

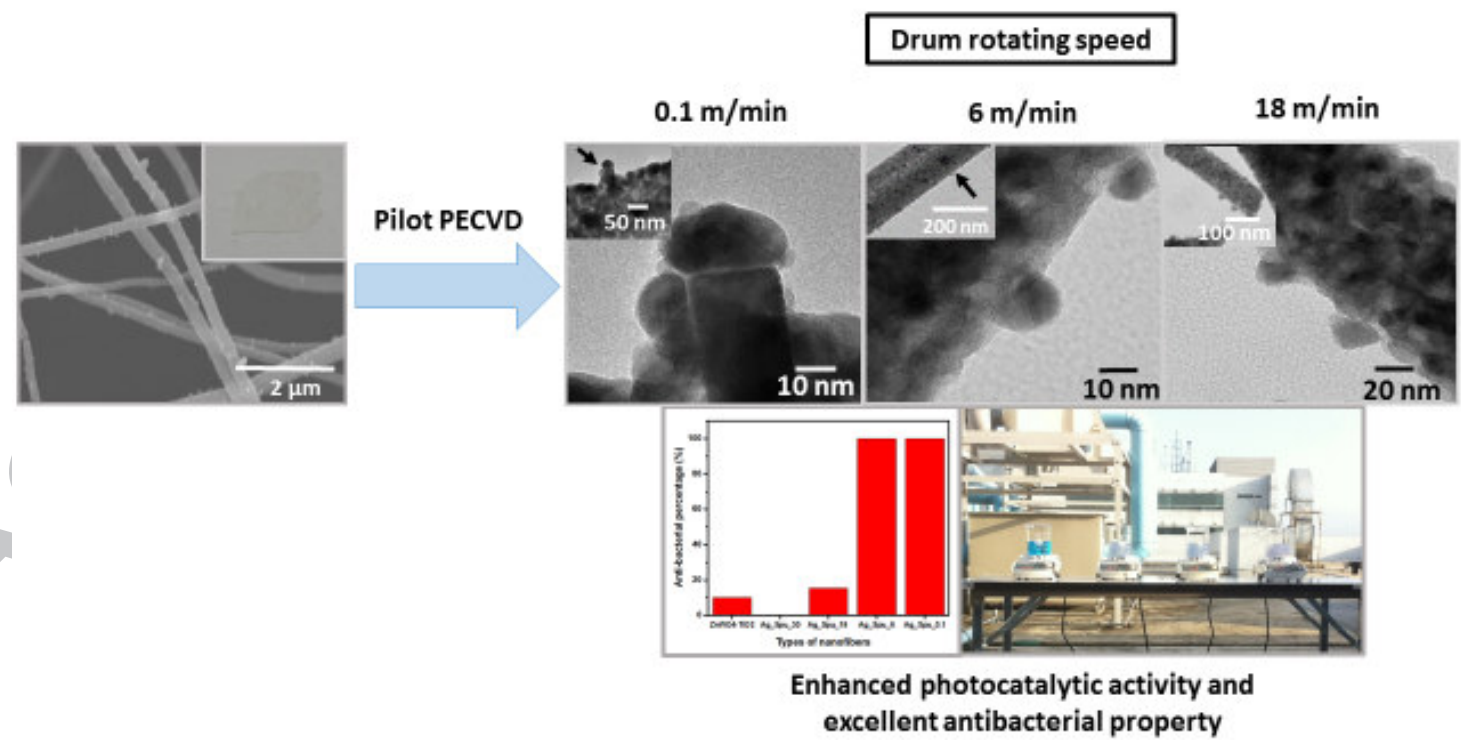

